\title{
3 Informed Consent and shared decision making in EBM
}

\section{1 'Informed consent' in regular medical practice}

'Consent' as it is understood in the medical context has to be asked from the patient and is the explicit agreement to waive a right to certain rules and norms which are normally expected in the treatment of other people and of ourselves as patients. Every surgical procedure would, without consent from the patient, be legally understood as assault and battery and the physician could be prosecuted for performing it. 'Informed consent' therefore in its most simple form means that the patient has received a good explanation about a medical procedure, understands what is happening to him or her and then can make an informed choice to accept or refuse, in the latter case the so called 'informed refusal. ${ }^{167}$ In order to give 'informed consent the patient has to be capable of understanding the information given by the physician. He or she must be competent to decide and to give consent voluntarily without being coerced by any means into giving consent. ${ }^{168}$ 'Autonomy' of the patient, hereby equated with 'person' plays the overarching role in 'informed consent.' A competent person who exercises autonomy will have the final say about their own life. 'Autonomy' itself is a contested term in the philosophy of science and interpretations therefore vary. According to Dworkin, "Liberty (positive or negative) ... dignity, integrity, individuality, independence, responsibility and self knowledge ... self assertion ... critical reflection ... freedom from obligation ... absence of external causation ... and knowledge of one's own interests." ${ }^{169}$ all fall under the definition of autonomy. For my argument I will stick with the definition of autonomy as being "aware of ones own interest" and the limitations that are set to the subsequent decisions by circumstances and societal norms. Decisions made by such an autonomous patient should be respected and adhered to. However "no theory of autonomy is acceptable if it presents an ideal beyond the reach of normal agents and choosers." 170 What Beauchamp and Childress mean by this is that no

167 Tom Beauchamp and James Childress. (2009). Principles of Biomedical Ethics. Oxford: Oxford University Press: 120.

168 Thomas Schramme. (2002). Bioethik. Frankfurt: Campus Verlag: 31.

169 Gerald Dworkin. (1988). The theory and practice of autonomy. Cambridge: Cambridge University Press: 6.

170 Tom Beauchamp and James Childress. (2009): 101. 
person is ever fully autonomous, since all persons are part of society, influenced by society and acting in accordance to it. All persons are dependent on something and especially in the medical context, patients are dependent on the information provided to them and on their own prior knowledge in order to make autonomous decisions as in refusing or consenting to a treatment on the basis of being thoroughly informed about it.

Again "informed consent has a role only where an activity is already subject to ethical, legal or other requirements." ${ }^{\prime 71}$ In today's medical practice, it is a usual procedure for the patient to give written consent before a surgery is performed. The surgeon explains the procedure, what is done, what is supposed to be the result and what are possible risks and side effects, and hands out written information that the patient normally can contemplate. Additionally to the surgeon, the anaesthetist and administrative staff also require informed consent signatures, since the patient and the clinic enter into a treatment contract. ${ }^{172}$ Unfortunately, the more people are involved, the more convoluted the information gets and the more confused some patients do become. Very often a large percentage of the information provided to the patient is written in medical language and does not help to enlighten a lay person. Therefore, paperwork concerning informed consent should be written in 'normal' language and the clinician should take the time to answer questions. If all informed consent papers are signed, the hospital administration will provide a contract between the hospital and the patient that allows for the procedure to take place.

\subsubsection{Implied or simple consent}

Patients can also give implied or simple consent, by allowing the physician to perform certain medical acts, such as drawing blood or giving a local anaesthesia (full anaesthesia needs written consent). Dentistry is a good example which often works without prior written consent. ${ }^{173}$ The sitting down and opening of the mouth is deemed to be implied consent for the procedure to be performed. This does not waive the necessity on the part of the dentist to inform the patient about the procedure ahead, possible risks and hopeful benefits. And the patient can revoke the consent at any time during the procedure.

171 Neil C. Manson and Onora O’Neill. (2007). Rethinking Informed Consent in Bioethics. Cambridge: Cambridge University Press: 72.

172 Andrew Lloyd, Paul Hayes, et.al. (2001). "The Role of Risk and Benefit Perception in Informed Consent for Surgery." in Medical Decision Making. 21(2): 141-149.

173 Kevin I. Reid. (2017). "Informed Consent in Dentistry." in The Journal of Law, Medicine and Ethics. 45(1): 77-94. 


\subsection{2 'Informed consent' and trust}

By giving 'informed consent,' a form of trust between the physician and the patient is established in which the patient allows for some kind of bodily invasion and trusts the physician that the intervention is in the patients' best interest. Informed consent in the medical setting is target-oriented. It is not a general waiver for the physician to do whatever he wants to do, but gives a limited ok for a certain procedure. Everything above and beyond that needs a new waiver, even if a patient is already under anaesthesia and needs to be woken up again to agree to the next procedure. And informed consent can never be understood as a fail-safe since it can be revoked at any time. So the physician has to be aware that even if the patient has consented to a procedure, it is the patients right to walk out of it. In reality, especially where surgeries are concerned, this case will hardly happen, but in medical research the participant can refuse to longer be a part of a trial and revoke the consent without the researcher having any recourse on the situation.

\subsubsection{Informed refusal}

A conflict between patient and physician can arise when a patient opts for 'informed refusal' and, for example, denies a life-saving intervention. The physician is required to protect life and to save it when in danger. The patient is formally allowed to not have certain procedures performed. ${ }^{174}$ In those cases where the patient is fully capable to make the decision to refuse treatment, the physician has to accept the patient's decision. These cases are most often prevalent in cancer treatments when a patient decides to stop the actual treatment and most often then opts for palliative care instead. The patient still has to sign a waiver stating that he will forgo active treatment, so that the physician is in the clear and cannot be legally prosecuted for not saving a life.

Many of these cases are uncontroversial. In many end-of-life decisions most clinicians agree to a change in treatment. The focus shifts from life-prolonging measures to palliative care where the 'quality of life' is judged more important. The main goal of a 'quality of life' care approach is to alleviate pain, fear and other symptoms, but to let nature run its course and to let the patient die with dignity. ${ }^{175}$ In palliative cases the patients wishes are paramount and it is acknowledged that the best possible evidence to treat the disease in question is not viable anymore for the patient in end-of-life care. However, there are cases where it is less clear why a patient is refusing treatment and where there might be even a conflict of interest

174 Thomas Schramme. (2002): 36.

175 Peter A. Singer, Douglas K.Martin, and Merrijoy Kelner. (1999). "Quality end-of-life care: patients' perspectives." in JAMA 13;281 (2): 163-168. 
between the patient and the physician. When a patient denies treatment out of religious reasons, most physicians will accept this, but if a patient denies lifesaving treatment because he or she is afraid of the procedure or of possible side effects then the physician has to aim at a better understanding of the situation on both sides. This understanding on the part of the physician is part of the 'informing process' to obtain 'informed consent' and to arrive at the ultimate goal of a shared decision. Conflicts of interest do arise when patients are refusing a life saving treatment without good reasons to do so. The physician has the obligation to save and protect life and has to react if and when the patient is in immediate danger. In some of these special cases the physician can and will question the ability and competence of the patient to refuse treatment. ${ }^{176}$

\subsubsection{Rare limitations to 'informed consent'}

There are very rare situations in medicine where the case is reversed. One such case has garnered enormous traction in the UK press over the summer of 2017. The terminally ill 11 month old baby boy, Charlie Gard, being treated at Great Ormond Street Hospital for mitochondrial depletion syndrome, was the focus of media attention for months. ${ }^{177}$ The disease in question is a cell-disease in which the mitochondria cease to produce the energy which the cells need to function. ${ }^{178}$ The hospital wanted to move the boy to palliative care and to let him die with dignity, while the parents wanted to take the boy to the USA to try a highly experimental treatment with a $10 \%$ chance of some change in the disease progression. The medication, nucleoside bypass therapy, is still in its very experimental phase and has not yet passed the laboratory stage. It has never been used on a patient with Charlie's specific strain and the US physician admitted that it would be highly unlikely that Charlie would actually benefit from the treatment. ${ }^{179}$ The parents and the hospital found themselves to be locked in a legal battle about who held the guardianship over the boy and who subsequently had the final say in treatment decisions. Even though these cases are fairly rare they do happen. In the UK, if such a case arises where the hospital and parents or carers are not agreeing on the treatment options, the courts have to make the last decision, not the parents and not the hospital. The parents in Charlie's case could not revoke the informed consent they had initially given to allow their son to be treated in the hospital. In the end and stressing here that the boy was terminally ill, the court decided against the

176 Thomas Schramme. (2002): 36.

177 Robert D. Truog. (2017). "The United Kingdom Sets Limits on Experimental Treatments: The Case of Charlie Gard." in JAMA. 318(11).

178 Josef Finsterer and Salma Wakil. (2015). "Mitochondrial Depletion Syndromes." in eLS. 1-9.

179 Robert D. Truog. (2017): 1001. 
parents wishes and the boy died in a hospice. In most other countries, it would have been possible for the parents to move their son to a different clinic, even to a different country. However, it is more than questionable if such a move would have changed the outcome in Charlie's case. The question in this case and in cases similar to these is not only about treatment options but also about the quality of life and how very important that aspect is in clinical medicine. Much more important than some critics of conventional medicine are giving the system credit for.

Above it is stated that 'informed consent' is target oriented and that a surgeon is not allowed to extend the 'informed consent' to perform an entirely different surgery. However, there are exceptions to this rule, when it can be deemed in the best interest of the patient to perform a lifesaving surgery, even though a different surgery was agreed upon. Such a special case falls under the category of emergency medicine. A vivid example of such a case was a young woman who was diagnosed with a tubal pregnancy which needed to be removed immediately to prevent further harm. During the surgery, the surgeon realised that the symptoms were actually not stemming from an ectopic pregnancy but from an acute appendicitis and that the pregnancy was actually in utero and intact. The surgeon decided to remove the appendix, thereby saving the patients life and the life of the unborn child. ${ }^{180}$ In this case the surgeon was legally 'allowed' to perform the altered surgery without obtaining prior informed consent from the patient. Acute appendicitis can lead to death and therefore it was an emergency situation. The risk to wake the patient up first was far greater because of possible complications due the acute appendicitis and due to the need to perform a second general anaesthesia in a very short time period, than to remove the appendix then and there.

In most emergency situations the overall goal is to save the patients life and at least to stabilise the patient until a hospital is reached. Emergency physicians often do not have the time to obtain 'informed consent', especially in case of accidents where multiple patients are involved and need to be treated. In many cases there is simply not the time nor the right situation to ask for the patients consent. In most cases it is assumed that the patient wants to be saved. It is a little different however when an emergency unit is called to a dying patient. Often families or carers all of sudden become afraid when a person is about to die and dial the emergency department in panic. In most of these cases it is hard to establish for the arriving team what the actual wish of the dying patient was to begin with and subsequently patients end up in hospital, on life support, without ever having consented to this kind of treatment. ${ }^{181}$

180 Kurt Hartman and Bryan Lang. (1999). "Exceptions to Informed Consent in Emergency Situations." in Hospital Physician: 54.

181 Roberto Forero, Geoff McDonnell, Blanca Gallego, et al. (2012). "A Literature Review on Care at the End-of-Life in the Emergency Department." in Emergency Medicine International. 


\subsection{The shift from paternalism to informed consent}

Before it became usual practice to ask for written consent by the patient, most physicians acted paternalistically. They decided what was best for the patient and acted accordingly, without explaining to the patient why it would be the best course of action and without pointing out alternatives or explaining that the patient does not have to agree to the intervention at all. ${ }^{182}$ As long as the physicians really had the best interest of the patient at heart, a paternalistic approach might not have been to the detriment of the patient, but the patient in this scenario is certainly lacking autonomy.

\subsubsection{From the Hippocratic Oath to the Declaration of Helsinki}

The Hippocratic Oath that most medical practitioners did, and in some form still do, swear since antiquity, and which was considered binding for the medical community, does forbid to harm the patient and/or to use the patient for the physician's gain. During the Second World War there were unfortunately many German physicians who disregarded the oath. They used concentration camp and prison inmates as guinea pigs, taking their deaths for granted and claiming they were doing so for the greater good of the German nation. During that time medical experimentation was not regulated, and even if it would have been regulated internationally, the NAZIS would have disregarded such regulations. There were no restrictions in place for the 'experimenters' to do whatever they deemed necessary. After the camps were liberated, and during the Nuremberg Trials, many of these atrocities came to light and triggered a movement to prevent such blatant disregard for the integrity and well-being of human beings in the future. ${ }^{183}$ Informed consent, given in a way as it is described above, plays a major role in this prevention program. Patients today can decide for themselves if, and often even when, they want to be subjected to medical procedures. They can choose the physician and they can refuse treatment, even though it might not be in their best interest. Nir Eyal describes informed consent as a tool to save trust in the medical profession. ${ }^{184}$ However, that trust can only be established if the tool of informed consent is used correctly. Written explanations, often in somewhat medical language, have to be supplemented with an active discussion between patient and physician in which questions can be

182 A discussion of the different forms of 'paternalism' would supersede the scope of this paper. For further reading: Thomas Schramme, Ed. (2015). New Perspectives on Paternalism and Health Care. Heidelberg: Springer Verlag.

183 Horst H. Freyhofer. (2004). The Nuremberg Medical Trial: The Holocaust and the Origin of the Nuremberg Medical Code (Studies in Modern History). New York: Peter Lang Publishing.

184 Nir Eyal. (2012). "Using Informed Consent to save trust." in JME Online. 
asked and treatment decisions can be made together. The physician has to recognise if the patient needs more time to make a decision, or if an initial discussion was sufficient to both parties to be comfortable with the treatment plan. The patient has to be aware that at any given point in time he or she has the possibility to stop the treatment and to either clarify procedures or to abandon a certain treatment altogether.

An even more important historical tool to achieve wide-spread trust in the medical profession was the Declaration of Geneva which is still in use today and followed the Nuremberg Code of 1947. The Nuremberg Code, conceived during and after the Nuremberg Trials, specified consent as 'voluntary consent' and demands that the subject giving consent cannot be coerced into doing so and knows what he is giving consent to. ${ }^{185}$ Manson and O'Neill point out that the Nuremberg Code does not exactly specify informed consent. The concept of patient autonomy or of consent with regard to later use of already acquired patient data or tissue is never mentioned or explained in detail in the Nuremberg Code. ${ }^{186}$ In order to clarify the points made in the Nuremberg Code, the World Medical Association ${ }^{187}$ came together in Geneva, Switzerland, and formulated eleven points which were hoped to represent a binding agreement for everyone in the medical profession. And thus was formulated for the first time the Declaration of Geneva. ${ }^{188}$

The Declaration of Geneva was first drafted in 1948 and was continuously amended over time, for the last time on October 14th, 2017. The Declaration of Geneva was and is understood, and used, as a modernised version of the Hippocratic Oath. But even the Declaration of Geneva only specifies the relationship between patient and physician, again without specifying informed consent and without taking medical research into account. But as has become already obvious, medical research transgresses the boundaries of medical practice. The patient-physician relationship collapses to a certain degree and transforms into a participantresearcher relationship. There will always be a conflict between the physician in the role as caregiver to the patient and the physician as researcher who needs participants for his research. The researcher cannot guarantee that his actions are in the patient's best interest. They might be beneficial for the actual patient, but they as well might not be. They certainly are beneficial for future patients, if all research

185 For the exact wording of the Nuremberg Code, please refer to https://history.nih.gov/re search/downloads/nuremberg.pdf. Last accessed on January $23^{\text {rd }}, 2020$.

186 Neil C. Manson and Onora O’Neill. (2007): 3.

187 The World Medical Association speaks for health care practitioners globally and continually strives to achieve and maintain better standards of medical care. To learn more about the World Medical Association, please refer to www.wma.net.

188 World Medical Association, The. "Declaration of Geneva." Adopted by the 2nd General Assembly of the World Medical Association, Geneva, Switzerland, September 1948. Lastly editorially revised by the WMA General Assembly, Chicago, United States, October 2017. 
is published, because either the new treatment is successful or it was abandoned due to harm and future patients are not exposed to it.

In order to codify and integrate medical research, the World Medical Association yet again came together and formulated the Declaration of Helsinki in 1964. The correct title of the Declaration is "Declaration of Helsinki - ethical principles for Medical Research involving Human Subjects." 189 Helsinki acknowledges the difficulty of the physician who is researcher at the same time. The first general principle sites the first point of the Declaration of Geneva. "The Declaration of Geneva of the WMA binds the physician with the words, "The health and wellbeing of my patient will be my first consideration, ${ }^{190}$ and the International Code of Medical Ethics ${ }^{191}$ declares that, 'A physician shall act in the patient's best interest when providing medical care". 192

Helsinki is supposed to set the standard for medical research ethics and is as such often used in national regulations or laws. However, the Declaration is not legally binding in international law. The latest version of Helsinki from 2013 includes 37 points and specifies and explains informed consent in medical research settings. In comparison to the version of 2008, the 2013 version has a whole paragraph titled "informed consent". The earlier version uses the term "competent human subjects" which is changed in the version of 2013 into "individuals capable of giving informed consent." The main aim of the paragraph about informed consent is not only to specify that it is important that consent is obtained, but also how encompassing the information needs to be and that the methods with which information is given should be fitting to the patient and not just a folder filled with medical terminology.

"In medical research involving human subjects capable of giving informed consent, each potential subject must be adequately informed of the aims, methods, sources of funding, any possible conflicts of interest, institutional affiliations of the researcher, the anticipated benefits and potential risks of the study and the discomfort it may entail, post-study provisions and any other relevant aspects of the study. The potential subject must be informed of the right to refuse to participate in the study or to withdraw consent to participate at any time without reprisal.

189 World Medical Association, The.WMA. "Declaration of Helsinki." Adopted by the 18th WMA General Assembly, Helsinki, Finland, June 1964. Lastly editorially revised by the 64th WMA General Assembly, Fortaleza, Brazil, October 2013.

190 World Medical Association. (2017). "Declaration of Geneva." 2nd paragraph.

191 World Medical Association, The. International Code of Medical Ethics from 1949. Adopted by the 3rd General Assembly of the World Medical Association, London, England, October 1949 and lastly amended by the 57th WMA General Assembly, Pilanesberg, South Africa, October 2006.

192 World Medical Association. (2013). "Declaration of Helsinki." Paragraph 3. https://www.wma. net/policies-post/wma-declaration-of-helsinki-ethical-principles-for-medical-research-involvinghuman-subjects/. Last accessed on January $23^{\text {rd }}, 2020$. 
Special attention should be given to the specific information needs of individual potential subjects as well as the methods used to deliver the information." ${ }^{193}$ The Declaration of Helsinki herewith acknowledges the distinction between medical practice and medical research and the shift in the patient/physician relationship.

\subsection{2 'Informed consent' and 'assent' for children, teens and physically or mentally incapacitated patients}

The whole chapter about 'informed consent' in the Declaration of Helsinki consists of eight paragraphs of which the one above is only the second and most elaborate. The following paragraphs specify special cases, such as when the patient is not capable of giving consent, as in children, teens or physically or mentally incapable persons. In these cases consent can be given by the parents or a "legally authorized representative." Being deemed incapable of giving informed consent however does not necessarily mean that the patients cannot voice their will at all. If the patient is able to give to understand the given information and form an opinion about it, he or she is deemed able to give 'assent,' for example teenager or mentally incapacitated patients. The investigator should in these cases get the patients assent or dissent in conjunction with the consent by a legal guardian. And the researcher should feel to be beholden to it the patients views. Consent, especially of teenagers, is often obtained without their knowledge, because it is deemed as being sufficient if and when the guardians are giving consent. ${ }^{194}$ Only when the patient is incompetent to give consent, either because he or she is in a coma or the handicap is too severe, then the investigator can forgo 'assent' but still has to obtain 'informed consent' by a legal guardian. The research has to "entail minimal risk and minimal burden" 195 and the reason why these patients need to be involved has "been stated in the research protocol and the study has been approved by a research ethics committee". ${ }^{196}$ Again, that point is extremely important, because during the Second World War, mentally incapacitated patients were deemed 'unworthy to live' [unwertes Leben] and were experimented on and killed without any ethical compunction by the researchers.

193 World Medical Asscoation. (2013). "Declaration of Helsinki." Paragraph 26.

194 World Medical Association. (2013). "Declaration of Helsinki." Paragraph 29.

195 World Medical Association. (2013). "Declaration of Helsinki." Paragraph 28.

196 World Medical Association. (2013). "Declaration of Helsinki." Paragraph 30. 


\subsection{The Tuskegee Experiment}

One rather spectacular case that transgressed the Declaration of Geneva and the Declaration of Helsinki is the Tuskegee Experiment. ${ }^{197}$

The Tuskegee Experiment started in 1932, hence before the Declaration of Geneva and well before the first Declaration of Helsinki, but in the time of segregation between black and white people in the South of the United States. It lasted until 1972. So instead of the initially proposed six months, the experiment lasted for 40 years. The experiment was initiated in and around Macon County, Alabama and conducted at the Tuskegee University. It was designed by the Public Health Service ${ }^{198}$ to observe the health effects of untreated syphilis in "black" men and was officially named "Tuskegee Study of Untreated Syphilis in the Negro Male." 399 men with syphilis and 201 without the disease were admitted to the study. The men were not informed of their health status but were only told that they were treated for 'bad blood', a term used to describe many, otherwise unspecified, illnesses. They were given some type of treatment to alleviate the symptoms and they were subjected to regular medical check-ups. Even when penicillin was recognised and became available as a cure against syphilis in 1947, these men did not receive the new treatment. ${ }^{199}$ The ethical and procedural problems of the Tuskegee experiment are manifold. First of all, the men randomised into the experiment were not informed of their health status and therefore could in no way give even some sort of informed consent to participate in the study. The benefits that were promised, like regular health check-ups, free meals and a burial insurance, coerced most of the men into participating since these were benefits which were otherwise unavailable for the participants. The area around Tuskegee was poor and the black population was even more so. The experiment was not designed to test a new drug to treat and/or cure syphilis but purely to show the overall effect of the disease if and when left untreated. In 1972 the experiment caused an outrage fuelled by different papers and was subsequently stopped a year later. An action - class law suit was filed and eventually settled out of court with a payment of 10 million Dollars. Survivors and their families received special benefits given to them by the government. ${ }^{200}$

197 Centres for disease control and prevention. "The Tuskegee Timeline." http://www.cdc.gov/tuske gee/timeline.htm Last accessed on January $23^{\text {rd }}, 2020$.

198 The Public Health Service (PHS) is a primary division of the United States Department of Health and Human Services. https://www.usphs.gov/. Last accessed on January $23^{\text {rd }}, 2020$.

199 Tuskegee University. National Centre for Bioethics in Research and Health Care. http://tuske geebioethics.org/ The usphs syphilis study can be found on this website, together with the historical apology and the information how the University of Tuskegee is coping with the legacy of the study. Last accessed on January $23^{\text {rd }}, 2020$.

200 Allan Brandt. (1978). "Racism and Research: The Case of the Tuskegee Syphilis Study." in Hastings Center Report, 8: 21-29. 
Those physicians and medical officials participating in the experiment violated at least five of the eleven points of the Declaration of Geneva and almost all points of the Declaration of Helsinki. The two most important violations in both are "the health of my patient will be my first consideration" and, only in the Declaration of Geneva, "I will not permit considerations of age, disease or disability, creed, ethnic origin, gender, nationality, political affiliation, race, sexual orientation, social standing or any other factor to intervene between my duty and my patient." ${ }^{201}$ The Tuskegee Experiment included only African American men in lower class circumstances without much education. The health of the patients was not the overall concern of the researchers. The were merely interested in the disease and its progression. Most of the men with syphilis were chosen because they were in the second and latent stage of the disease. Some historians seem to think that this fact makes the experiment a little less questionable, because these men might not have benefitted from penicillin at all and they could not pass on the disease anymore. Realistically, syphilis in its second or latent stage is symptom free and, after about one year, cannot be passed on anymore. However, following the NHS syphilis guide, syphilis can successfully be treated in the latent stage with antibiotic medication, such as penicillin. ${ }^{202}$ The excuse given by some historians and those researchers who proposed to continue the experiment therefore was not valid. Even though the disease in its second stage is tolerable to live with, if it moves into the third stage it is most often, if not always, deadly.

Basically these men were treated as a form of lab animal. Some were not yet in the latent stage or in the early latent stage, and did pass on the disease. All participants were discouraged to seek medical help outside the study. When the patients and the wider public were informed of the obvious maltreatment, the previously established trust between the researchers and the patients was irreversibly broken. And unfortunately that breeching of trust has its repercussion in the African American Community until today.

\subsubsection{Consequences of Tuskegee}

Most studies, especially in the USA where minority groups are so prevalent, try to have as wide a cross-section through the population as possible and therefore try to involve as many members of minority groups as possible. Although advertisements of trials are often especially designed as to reach these communities, participation is minimal. In almost every paper concerning itself with this problem, the

201 World Medical Association. (2017). "Declaration of Geneva." Paragraph 5.

202 National Health Service, UK. NHS Choices: your health, your choices. http://www.nhs.uk/Con ditions/Syphilis/Pages/Treatmentpg.aspx Last accessed on January $23^{\text {rd }}, 2020$. 
Tuskegee Experiment is cited as the negative example for a study that is still prevalent in the minds of the people. ${ }^{203}$ Most minority groups in the USA seem to not know the difference between a clinical experiment and a clinical study and believe that they will be treated as guinea pigs for the white people. ${ }^{204}$ It might sound like semantics, but the difference between 'experiment' and 'study' is quite important. Experiments should be strictly confined to the laboratory setting and not involve human beings on the receiving end. Studies are conducted outside the laboratory and are so-called first-in-man studies.

\section{4 'Informed consent' in clinical research}

One of the pledges of both Declarations that was so irretrievably broken in the Tuskegee Experiment, is "The health of my patient will be my first consideration." In 'normal' clinical practice, this pledge seems to be easy enough to maintain. In clinical research, the health of 'the patient as such' is also the first consideration. But, since new procedures or medications are developed, researchers cannot be sure if the actual patient will benefit from their research. Even though they might believe their procedure to be more effective, it might not be and worse it might even be dangerous. The danger of adverse effects is one of the main risks patients need to be informed about. Today, clinical research is conducted in a way that differs widely from earlier 'experiments'. It is under strict regulations and a trial involving human beings is only allowed after rigorous testing in the laboratory has happened. "Medical research involving human subjects must conform to generally accepted scientific principles, be based on a thorough knowledge of the scientific literature, other relevant sources of information, and adequate laboratory and, as appropriate, animal experimentation. The welfare of animals used for research must be respected." 205 Danger to the participants is supposed to be minimised as much as possible. And the participant has to understand the difference between clinical practice and clinical research and be aware that in a research setting his status has changed from a regular 'patient' to a 'participant.' Accordingly, the physician changes into a researcher, and both parties, participant and researcher should be aware of that and adjust their expectations accordingly. ${ }^{206}$ The researcher does not automatically act in the patients' best interest anymore. He or she is well aware that there might be unknown dangers in the proposed procedure

203 Vicki S. Freimuth and Sandra Crousse Quinn, et.al. (2001). "African Americans' views on research and the Tuskegee Syphilis Study." In Social Science and Medicine 52: 2.

204 Vicky S. Freimuth and Sandra Crousse Quinn, et.al. (2001):

205 World Medical Association. "Declaration of Helsinki." (2013). Paragraph 21.

206 Robert D. Truog. (1999). "Informed Consent and research design in critical care medicine." in Critical Care. 3(3). and Manson and O’Neill. 
or drug. Therefore it seems to be important that researcher and participant understand each other as two parts of a research team which is operating outside the usual clinical practice. If there is an actual benefit for the participant involved, then this benefit should be understood as a form of positive side-effect, but not as an expected and predictable outcome. Although clinical research by its very nature is aiming to produce positive effects, there can be no guarantee that it does so. For the actual research, negative effects are equally valid and informative, especially in preventing harm for future patients.

\subsubsection{Use of already established data in and for research}

In some cases, trials or observational studies have been performed but the data has not been used when it was amassed. The question which needs to be asked then is if the available data can be used for further research without the consent of the patients? And what to do with the data if consent is not possible to obtain or if the patients refuse to give it? This question can also pertain to health data that is amassed during regular practice. Is it allowable to use regular patient data, without the patients consent, for research? Just to make it clear, data here is never personal data which is in almost all cases strictly confidential. The data we are talking about here is anonymised data without disclosure about the actual patient. Following Goldacres' idea, discussed in a previous chapter, of a large randomised trial for different statins for example, it would be absolutely enough for the researcher to have basic, anonymised data available containing gender, age, and weight of the patient, the type of statins prescribed and the reason for the prescription. ${ }^{207}$ The report about positive and negative effects of the treatment and if other medication had been taken at the same time and potentially interacted with the statin would also be included. This information would be impossible to trace back to a single individual patient. The question would be if it would be necessary in this example to obtain informed consent? There is no agreed upon standard available in medicine to ultimately decide this question. Some argue that it in every case in which any kind of data is used, informed consent has to be acquired. Some argue that if and when the data is sufficiently anonymised it is allowable to use it for the greater good of the community and as part of the social welfare system. Especially were the adoption of health policies is concerned. ${ }^{208}$

207 Goldacre, Ben. (2012): 225.

208 Onora O'Neill. (2003). "Some limits of informed consent." in Journal of Medical Ethics. 29: 4-7. 


\subsubsection{The issue of trust revisited}

Using data without the informed consent of the patient can lead to an overall mistrust of the population into the wider medical community. Manson and O'Neill argue that we "need to build and maintain trust as reason to demand informed consent for clinical care." 209 One reason for this is, and here I am following Eyal, that the "value of mutual trust is a contributor to population health." ${ }^{10} \mathrm{Her}$ argument is that if there is an overall trust in the medical services within the population, then people are more willing to consult the medical community or to participate in medical trials. If there is an overall mistrust, as there is in the African American community, Eyal also mentions Tuskegee in her paper, then the overall willingness to seek treatment is lower and the overall population health is worse in comparison to more trusting communities. In order therefore to have patients as well informed and willing participants in a medical trial, there has to be an overall trust in the medical community. Eyal's argument sounds compelling, however, it does not provide a solution for establishing trust, other than saying that it needs to be won through information and explanations.

In western countries the medical community however seems to be loosing the trust of the population, at least in parts. Many patients are dissatisfied with the overall medical treatment, suffer from the time constraints that most physicians are under and do not care about the medical information available. A case in point here is the overall popularity of homeopathic treatments and the refusal to vaccinate children. ${ }^{211}$ Especially parents with a higher education and a higher income increasingly refuse to follow the advise of the WHO for vaccinations. Reasons for this refusal are manifold. Some claim that the vaccine against mumps, measles and rubella causes autism. A claim initially made by Andrew Wakefield, MD, which has been debunked by the medical community and which led to Dr. Wakefield to not longer be allowed to practise medicine in the UK. ${ }^{212}$ Another reason which is often used is that these children's diseases are needed for the healthy build-up of the immune system and that vaccines are more dangerous than possible complications of these diseases. Again a myth which has been debunked by the medical community but which is widely upheld in lay circles. ${ }^{213}$ To reestablish the trust in the medical community for these parents would first and foremost mean to actually

209 Neil C Manson and Onora O’Neill. (2007):

210 Nir Eyal. (2012): 1.

211 I have devoted an entire chapter to the problems and pitfalls of homeopathy.

212 Fiona Godlee, Jane Smith, and Harvey Marcovitch. (2011). "Wakefield's article linking MMR vaccine and autism was fraudulent." in BMJ 342.

213 Daniel A. Salmon, Matthew Z. Dudley, Jason M. Glanz, and Saad B. Omer. (2015). "Vaccine Hesitancy Causes, Consequences, and a Call to Action" in American Journal of Preventive Medicine and Elsevier. 
reach them and to provide convincing information. But since medicine and health today has sort of reached the status of a religion, it is not on a scientific basis that those who do not believe in actual medicine can be reached. The problem of informed consent and trust in population health is therefore divided into two distinct problems. The first problem is to maintain the trust in those who use mainstream, or conventional medicine. Here information, based on evidence, is the key. The information must be complete, yet again that is why all data is needed, and needed to be published, to make a decision, and the physician has to aim at explanations of the available evidence in terms that are understandable to the lay person. The second problem is to build up trust in those who are sceptical of conventional medicine. Here evidential scientific information is not enough. It is still the basis which is needed to convince people but it seems as if the convincing has to happen on an emotional level. However, I fail to see a solution how those sceptics can be reached at all.

\subsection{How to transmit medical information successfully}

An important question that seems to me to be often overlooked in the literature about informed consent is how to actually achieve the 'informed' part. The guidelines are rather vague. The researcher is supposed to explain the procedure or medication, its benefits, side effects and risks and in case of a placebo-controlled trial, the researcher should also make clear that those receiving the placebo are actually not receiving any medication at all. Those who are deemed to be able to give consent are deemed to be able to fully understand that information and to make their decision accordingly. To reach the 'informed' part therefore, some type of exchange between the researcher and the participant has to have taken place. ${ }^{214}$ This presupposes however, that both parties are speaking the same language and use and understand the same terminology. The researcher has to phrase the medical information in such a way that the lay person can make educated deductions and understand what he is told. The researcher also has to take the social background of the patient into account. If the information were couched in purely medical terms, then the lay person probably will gain little knowledge from the exchange and although it can be stated that he has been informed, it is doubtful if the consent that is subsequently given can be understood as 'informed' consent. The patient might agree out of trust, even though that trust is not based on information and therefore also not on the act of informing, but on a personal feeling. And personal feelings can also play a huge part in refusing a treatment, as in the case of those refusing vaccinations.

214 Neil Manson and Onora O’Neill.(2007): 42. 
Manson and O'Neill argue that since the act of informing is based on communication, information can also be conveyed by non-verbal communication. ${ }^{215}$ Non-verbal communication can lead to the establishing of trust without the necessity that the verbal communication is understood. This trust can lead to a form of selection bias that subsequently can influence the outcome of the trial. Informed consent, trust and selection bias on the part of the participant are therefore intertwined. Selection bias can lead the participant to the 'false' conclusion that the care he is receiving is superior to the standard care. This positive feeling can provoke a feeling of well-being in the patient, even though he might receive 'only' a placebo. However, selection bias can be prevented in trials through sufficient blinding of all participants. Since a blinded physician can only convey what he or she knows about the trial and not in which arm the participant is randomised into, even though the participant might be trusting, he or she cannot base the trust on any information that is not known.

The available information needs to be transmitted in a way that the patient or participant can understand it, can question it, and is subsequently able to base a decision on it. For that purpose the information should not only be written but also transmitted verbally so that the physician is able to make amendments to the information based on the patients/participants questions and reactions.

\subsection{Conclusion}

Informed consent is an important tool for both medical research and medical practice. It can and should be used as a patient/participant safe-guard and as a needed process in shared decision making. The information should be provided in written and verbal form so that the patient/participant has, in the former case, the time to read the information and to formulate questions, and in the latter case, to ask the questions and to seek clarifications.

However, the limits of informed consent should be acknowledged. There are cases, as we have seen, were informed consent would be deemed necessary but cannot be freely given because of limitations on the part of the patient. However, a lack of informed consent does not automatically prevent medical interference to, for example, save a patient in an emergency situation. A lack of informed consent should also not prevent the use of data for the establishing of health policies. The emphasis in this case should be on the use of data, not the use of humans as research objects. The latter case always and under any circumstances needs informed consent by the person. Informed consent therefore is an important tool in medical research which leads to greater understanding for both, patients and researchers.

215 Neil Manson and Onora O’Neill. (2007): 55. 
In this way, informed consent is useful because it can promote knowledge, since the need to explain a procedure inevitably leads to greater understanding. And greater understanding can lead to the advance of medical knowledge. As we have seen however, medical research does not inevitably lead to greater medical knowledge. RCTs do not lead to knowledge, they lead to evidence and evidence can sometimes be severely flawed. Therefore, the next chapter will deal with the question why it is called evidence-based medicine and not knowledge-based medicine and where the differences are.

Open Access This chapter is licensed under the terms of the Creative Commons Attribution 4.0 International License (http://creativecommons.org/licenses/by/4.0/), which permits use, sharing, adaptation, distribution and reproduction in any medium or format, as long as you give appropriate credit to the original author(s) and the source, provide a link to the Creative Commons license and indicate if changes were made.

The images or other third party material in this chapter are included in the chapter's Creative Commons license, unless indicated otherwise in a credit line to the material. If material is not included in the chapter's Creative Commons license and your intended use is not permitted by statutory regulation or exceeds the permitted use, you will need to obtain permission directly from the copyright holder.. 